\title{
ESTUDO COMPARATIVO NÃO RANDOMIZADO DO MIDAZOLAM VERSUS THIOPENTAL EM CRIANÇAS COM ESTADO DE MAL EPILÉTPTICO REFRATÁRIO
}

\author{
ALFREDO LÖHR JUNIOR*, LINEU CESAR WERNECK ${ }^{\star *}$
}

RESUMO - Avaliamos o uso do midazolam em relação ao thiopental em crianças com estado de mal epiléptico refratário(EMER). Foram estudadas 50 crianças com EMER, internadas em UTI-pediátrica, composto pelos: Grupo Midazolam(A) (n=24) prospectivo, e o Grupo Thiopental(B) (n=26) histórico. Benzodiazepina e difenil-hidantoina e outros anticonvulsivantes foram previamente utilizadas nos dois grupos. Não havendo o controle das convulsões, no Grupo A os pacientes receberam uma dose de $200 \mu \mathrm{g} / \mathrm{kg}$ midazolam intravenoso em bolo, seguindo-se a infusão intravenosa contínua na razão de 0,25 a $15 \mu \mathrm{g} / \mathrm{kg} / \mathrm{min}$. No grupo B foi administrado thiopental, uma dose em bolo intravenosa de $1 \mathrm{mg} / \mathrm{kg}$,, seguida de infusão intravenosa contínua na razão de 10-120 $\mu \mathrm{g} / \mathrm{kg} / \mathrm{min}$. Em relação ao tempo de controle das crises e sua eficácia, não houve significância estatística para os dois grupos. No Grupo A houve significativamente menos complicações durante o tratamento: menos cianose $(\mathrm{p}=0,00006)$ e menos crianças necessitaram de ventilação assistida ( $\mathrm{p}<0,00001)$. No Grupo A, $12,5 \%$ dos pacientes, na pós-terapia imediata, apresentaram alterações psíquicas, como agitação, confusão mental, alucinação visual.

PALAVRAS-CHAVE: midazolam, estado de mal epiléptico, tratamento.

Comparative non-randomized study with midazolam versus thiopental in children with refractory status epilepticus

ABSTRACT - We evaluated the use of midazolam versus thiopental in 50 children with refractory status epilepticus (RSE), admitted in a pediatric intensive care unit. The study consisted of two groups of patients: Group A - Midazolam, a prospective study, and Group B - Thiopental, a historical group. These patients already had previous medication with benzodiazepin and diphenylhydantoin and other drugs. When there was no effective control of the seizures, the patients of Group A received midazolam of $200 \mu \mathrm{g} / \mathrm{kg}$ intravenous in bolus, being followed by continuous intravenous infusion at the rate $0.25-15 \mu / \mathrm{kg} / \mathrm{min}$. Group B received thiopental $1 \mathrm{mg} / \mathrm{kg}$ intravenous in bolus followed by continous intravenous infusion at the rate of $10-120 \mu \mathrm{g} / \mathrm{kg} / \mathrm{min}$. In relation to the time of seizure control and effectiveness, there was no statistical significance for the two groups. The Midazolam Group had significantly less complications during the treatment: less cyanosis ( $\mathrm{p}=0.00006)$, and they did not need respiratory support $(\mathrm{p}<0.00001)$. When the therapy with midazolam was ceased, $12.5 \%$ of the patients from this group showed psychological disorders such as mental confusion, aggressive behavior, restlessness, hallucinations and agitation.

KEY WORDS: midazolam, status epilepticus, treatment

O estado de mal epiléptico (EME) representa frequentemente uma emergência neurológica em pediatria. O EME é definido como qualquer tipo de crise epiléptica única ou recorrente com duração superior a 30 minutos, durante as quais não há recuperação do nível de consciência ${ }^{1,2}$. Quando o EME ultrapassa os 60 minutos, é denominado refratário (EMER) ${ }^{3}$. O EME pode ser determinado por insulto agudo no sistema nervoso central, ou manifestar-se secundariamente a

*Professor Adjunto do Centro de Ciênicas Biológicas e da Saúde da Pontifícia Universidade Católica do Paraná, Coordenador do Serviço de Neurologia Infantil do Hospital Infantil Pequeno Príncipe, Curitiba-Paraná; **Professor Titular de Neurologia do Departamento de Clínica Médica e do Serviço de Doenças Neuromusculares do Hospital de Clínicas, Universidade Federal do Paraná, Curitiba-Paraná. Aceite: 18-janeiro-2000.

Dr. Alfredo Löhr Jr - Hospital Infantil Pequeno Príncipe - Rua Dez. Motta 1100 - 80250-060 Curitiba PR Brasil.E mail: fredd@rla01.pucpr.br 
epilepsia sintomática remota. O EME é classificado como convulsivo e não convulsivo, sendo a forma convulsiva predominante e mais grave ${ }^{4}$. A mortalidade pelo EME está geralmente mais associada com a sua etiologia e as suas complicações, do que ao tempo de atividade epiléptica "per se". A taxa de mortalidade segundo a literatura situa-se entre $3 \%$ e $53 \%$. A difenil-hidantoína, os barbitúricos, assim como os benzodiazepínicos, são as drogas eleitas na atualidade para o tratamento do EME. Com relação aos benzodiazepínicos têm sido publicados alguns estudos, entre eles destacando-se os de Rivera e colaboradores e Koul e colaboradores, preconizando os autores o uso de midazolam por infusão contínua em crianças no controle do $\mathrm{EME}^{6-8}$.

Considerando-se a falta de consenso sobre o uso do midazolam no EME em crianças do nosso meio, procurou-se avaliar a eficiência do uso desta droga por infusão contínua intravenosa, em crianças com EME do tipo convulsivo generalizado e parcial. Paralelamente, o estudo procurou também estabelecer a comparação da eficiência do midazolam em relação ao thiopental por infusão contínua intravenosa empregado em outro grupo de crianças.

\section{MÉTODO}

Foram estudados 2 grupos de crianças com o diagnóstico clínico de EMER, tipo convulsivo, generalizado e parcial, que foram internadas na Unidade de Terapia Intensiva (UTI) do Hospital Infantil Pequeno Príncipe (HIPP), Curitiba, Paraná, Brasil, no período de abril de 1990 a novembro de 1997. O estudo abrangeu dois grupos: Grupo Midazolam (Prospectivo) e Grupo Thiopental (Histórico). O Grupo Midazolam foi composto de 24 casos; 4 pacientes desse grupo entraram no estudo duas vezes, em função da recorrência do EME, após alguns dias. Este grupo foi constituído de 11 crianças do sexo masculino e 13 do sexo feminino e com idade variando de 3 meses a 17 anos, com a mediana de 3 anos e 4 meses, (apenas um caso com 17 anos, porém, com um desenvolvimento pondo-estatutural compatível com 7 anos). O peso variou de 3,8 a $45 \mathrm{~kg}$ com a mediana de 14,5 kg. O Grupo Thiopental foi composto por 26 casos, sendo 11 do sexo masculino e 15 do sexo feminino e com a idade entre 2 meses e 12 anos, mediana de 10 meses. O peso variou de 6,6 a $26 \mathrm{~kg} \mathrm{com}$ a mediana de $8,0 \mathrm{~kg}$.

Critérios de inclusão - Pacientes com EME, com caracterização clínica de crise convulsiva contínua ou subentrante, com alteração ou perda da consciência e com duração igual ou superior a 60 minutos ${ }^{3,9,10}$. Todos os pacientes receberam previamente benzodiazepina $0,3-0,5 \mathrm{mg} / \mathrm{kg}$, administrada por via intravenosa ou intramuscular em uma ou duas doses, e seguindo-se o uso de fenitoína $15-20 \mathrm{mg} / \mathrm{kg} /$ dose por via intravenosa. Com relação ao Grupo Midazolam, por questões éticas, 21 pacientes continuaram a receber outros anticonvulsivantes, listados na Tabela 1, os quais vinham sendo usados no momento em que se instalou a infusão contínua do midazolam. Os tipos de crises epilépticas que caracterizaram o EME, sua frequência e duração foram observados e anotados em intervalos de 6 horas, pelo médico intensivista da UTI e registrados na ficha de evolução médica destinadas para este fim. No Grupo Thiopental, os dados foram obtidos dos prontuários médicos. Os critérios de inclusão obedeceram as mesmas normas do Grupo Midazolam. No grupo Thiopental, nove pacientes continuaram a receber outros anticonvulsivantes, listados na Tabela 1, quando foi instalado o thiopental por infusão intravenosa contínua.

No Grupo Midazolam, uma vez caracterizados os pacientes com EME, pelo médico do pronto-atendimento, e ou do ambulatório de emergência, esses foram transferidos para UTI do HIPP. Foram monitorizados através de monitor cardíaco e o oxigênio controlado através do oxímetro de pulso. O aporte de oxigênio foi oferecido conforme a necessidade, por máscara, catéter nasal ou entubação. A hidratação, a correção dos distúrbios hidroeletrolíticos, metabólicos e a antibioticoterapia foram executadas de acordo com a necessidade de cada paciente, julgada pelo médico intensivista de plantão. Procederam-se os exames complementares que seguem. Antes do início da terapia com o midazolam: hemograma, sódio, potássio, cálcio, gasometria e glicemia. No decorrer da internação foram realizados, entre o $1^{\circ}$ e o $4^{\circ}$ dia de internação o eletrencefalograma tomografia axial computadorizada do crânio e de acordo com a necessidade e para determinar a investigação complementar quanto a etiologia do EME: alanino e asparto aminotransferase, amônia, ácido láctico, uréia, creatinina, coagulograma completo, tempo de protombina, líquido cefaloraquidiano (citologia, bioquímica, imunologia), hemocultura, urocultura, triagem urinária para erros inatos do metabolismo e toxicológico, radiografia de tórax, biópsia hepática ou de medula óssea.

Considerados todos os procedimentos anteriormente descritos e após os pacientes receberem outras drogas antiepilépticas, foi administrada uma dose inicial de midazolam $200 \mu \mathrm{g} / \mathrm{kg}$, por via intravenosa em bolo, em 3 a 4 minutos. Na sequência, foi usado midazolam, em infusão contínua por via intravenosa, na razão de $0,25 \mu \mathrm{g} / \mathrm{kg} /$ minuto a $15 \mu \mathrm{g} / \mathrm{kg} / \mathrm{minuto}$ utilizando-se bomba de infusão. Conforme a necessidade, dobrou-se a dosagem da infusão entre 30 minutos a 3 horas até se obter o controle clínico das crises, sendo mantido por um período mínimo de 6 horas e reduzindo-se a seguir, na mesma razão da utilizada durante introdução do medicamento. 
Tabela 1 - Uso concomitante de outros anticonvulsivantes à terapia dos grupos midazolam e thiopental.

\begin{tabular}{lcccc}
\hline Tipo de anticonvulsivante & \multicolumn{2}{c}{ Grupo A (n=24) } & \multicolumn{2}{c}{ Grupo B (n=26) } \\
& Número & $\%$ & 17 & Número \\
\hline Não usado & 3 & 12,5 & 06 & 65,4 (p=0,0004 \\
Difenil-hidantoina & 6 & 25,0 & - & - \\
Lamotrigina & 1 & 4,2 & - & - \\
Ácido valpróico & 2 & 8,3 & 03 & 11,5 \\
Fenobarbital+difenil-hidantoina & 6 & 25,0 & - & - \\
Prednisona+valproato+benzodiazepina & 1 & 4,2 & - & - \\
Fenobarbital+carbamazepina & 2 & 8,3 & - & - \\
Vigabatrina+valproato+difenil-hidantoína & 2 & 8,3 & - & - \\
Fenobarbital+nitrazepam & 1 & 4,2 & & \\
\hline
\end{tabular}

Grupo A, Midazolam; Grupo B, Thiopental.

O Grupo Thiopental foi constituído de 26 pacientes, sendo os tipos e a duração das crises, coletados através de análise dos prontuários médicos dos pacientes internados na UTI do HIPP. Todos os pacientes deste grupo foram submetidos à mesma conduta, no tocante à medicação pré-thiopental e às normas para solicitação dos exames complementares. Os cuidados com a monitorização cardíaca e a oximetria foram semelhantes aos do Grupo Midazolam. A oxigenioterapia, o controle dos distúrbios hidroeletrolíticos, metabólicos, hidratação, antibioticoterapia e demais cuidados gerais e intensivos foram idênticos aos do Grupo Midazolam.

Considerados todos os procedimentos anteriores, os pacientes do Grupo Thiopental, receberam o thiopental, conforme a literatura ${ }^{11,12}$, inicialmente a dose de $1 \mathrm{mg} / \mathrm{kg}$ por via intravenosa em bolo, diluido em solução fisiológica. A seguir, o thiopental foi diluído em solução fisiológica por via intravenosa com bomba de infusão contínua, na dose inicial de $10 \mu \mathrm{g} / \mathrm{kg} /$ minuto, dobrando-se em média a dose a cada 30 a 60 minutos, até o máximo de $120 \mu \mathrm{g} /$ $\mathrm{kg} / \mathrm{minuto}$, até a obtenção do controle clínico das convulsões. O thiopental foi retirado gradualmente após o mínimo de 6 horas de controle das convulsões.

O estudo foi realizado no Grupo Midazolam com a autorização da Comissão de Ética Médica do HIPP, e com o consentimento informado por escrito do responsável pelos pacientes. No Grupo Thiopental, em se tratando de análise de prontuários, o consentimento foi dado pela Comissão de Ética do HIPP.

Recorreu-se à análise estatística de comparação dos dados, sendo utilizados os testes t de Student para grupos dependentes, e os não paramétricos Mann-Whitney. Ambos através do Software "Primer of Biostatics", "Qui-Quadrado com correção de Yates" e "Exato de Fischer" (Através do software "Epi-Info") para o caso de amostras independentes.

\section{RESULTADOS}

A etiologia nos dois grupos foi considerada de acordo com a classificação etiológica proposta por Shinnar e col. ${ }^{13}$, como: sintomático agudo, sintomático remoto, idiopático, idiopático febril e encefalopatia progressiva, e os achados estão demonstrados na Figura 1. Os tipos de crises epilépticas que predominaram no Grupo Midazolam foram: tônica generalizada, clônica parcial e tônica parcial. No Grupo Thiopental predominaram: a tônica generalizada, clônica generalizada e parciais complexas (Fig 2). Com relação à necessidade de ventilação assistida, somente 3,8\% dos pacientes do Grupo Midazolam necessitaram de suporte respiratório, enquanto que no Grupo Thiopental 96,2\% foram ventilados mecanicamente ( $\mathrm{p}<0,00001)$. A dose inicial mínima de infusão intravenosa do midazolam foi $0,25 \mu \mathrm{g} / \mathrm{kg} / \mathrm{min}$ e a dose máxima foi de $15 \mu \mathrm{g} / \mathrm{kg} / \mathrm{min}$. A dose média de infusão contínua para o controle das crises foi $2,0 \mu \mathrm{g} / \mathrm{kg} / \mathrm{min}$., equivalente à $0,12 \mathrm{mg} / \mathrm{kg} / \mathrm{hora}$. Para o thiopental, a dose mínima foi $10 \mu \mathrm{g} / \mathrm{kg} / \mathrm{min}$. e a máxima $120 \mu \mathrm{g} / \mathrm{kg} / \mathrm{min}$., sendo a média de $41 \mu \mathrm{g} / \mathrm{kg} / \mathrm{min}$. (2,46 mg/kg/hora).

O Grupo Thiopental teve controle das crises em maior número de pacientes e em menor tempo em relação ao Grupo Midazolam (Fig 3).

Considerando as complicações clínicas, houve dados estatisticamente significativos referente a presença de pneumonia, sendo de $8 \%$ dos casos para o Grupo Midazolam e $42 \%$ para o Grupo 


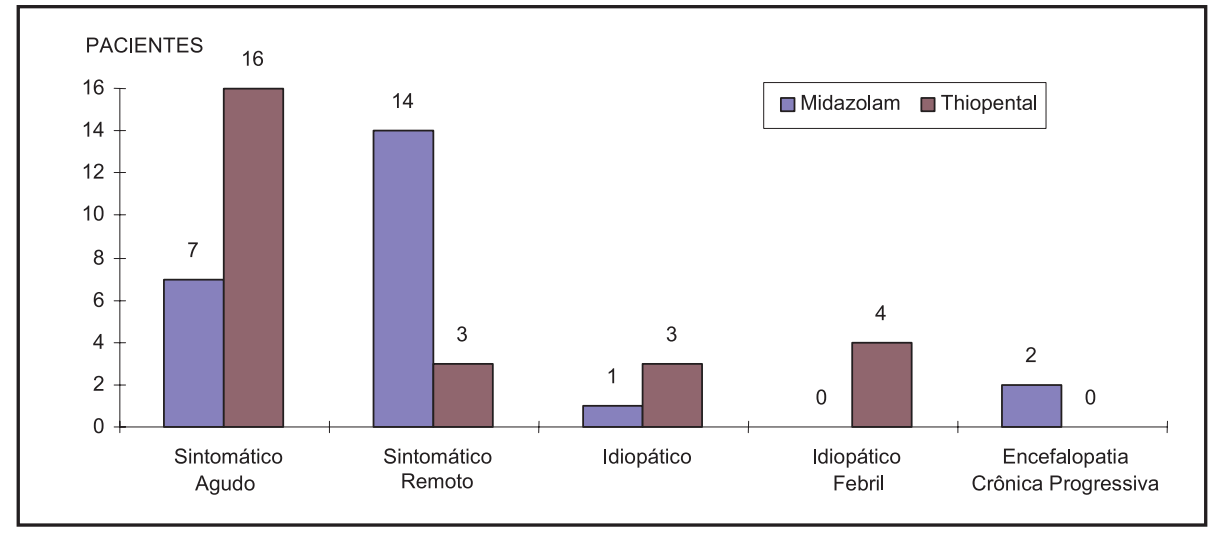

Fig 1. Etiologia observada nos pacientes medicados com midazolam e thiopental.

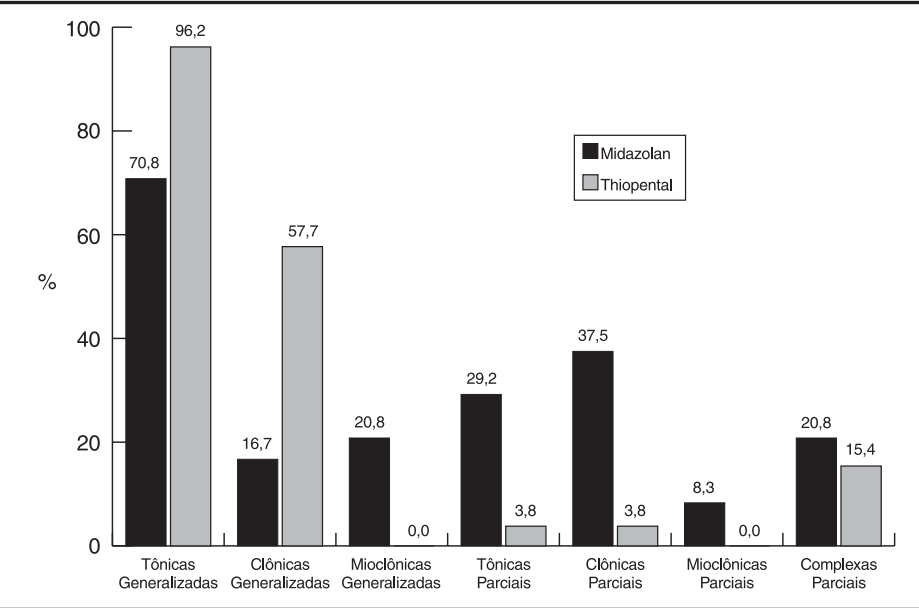

Fig 2. Descrição das crises observadas nos pacientes dos grupos de midazolam e de thiopental.

Thiopental ( $\mathrm{p}=0,0158)$. A cianose de extremidades e região peri-oral se fez presente em $61,5 \%$ no Grupo Thiopental e ocorreu só em 4,1\% dos pacientes do Grupo Midazolam ( $\mathrm{p}=0,0006)$. A ocorrência de acidose metabólica na admissão foi de 4,2 \% no Grupo Midazolam e 46,2\% no Grupo Thiopental e durante as terapias foi de 8,3\% para o Grupo Midazolam e 30,8\% para o Grupo Thiopental. Não foram encontradas alterações estatisticamente significativas entre os grupos nas alterações dos eletrólitos (cálcio, sódio e potássio) na admissão e durante as terapias. Não foi detectada qualquer crise de ausência típica, eletricamente demonstrável nos dois grupos.

A taxa de mortalidade dentro período de internação, foi 15\% no Grupo Midazolam e 19,2\% no Grupo Thiopental. No Grupo Midazolam, foram observados agitação psicomotora importante, agressividade e alucinações visuais, imediatamente após o término do uso do midazolam em três pacientes com idade superior a 2 anos.

\section{DISCUSSÃO}

A distribuição do gênero foi uniforme nos dois grupos. No entanto, não há concordância entre os autores no tocante a equivalência dos sexos nesta emergência neurológica. Phillips \& 


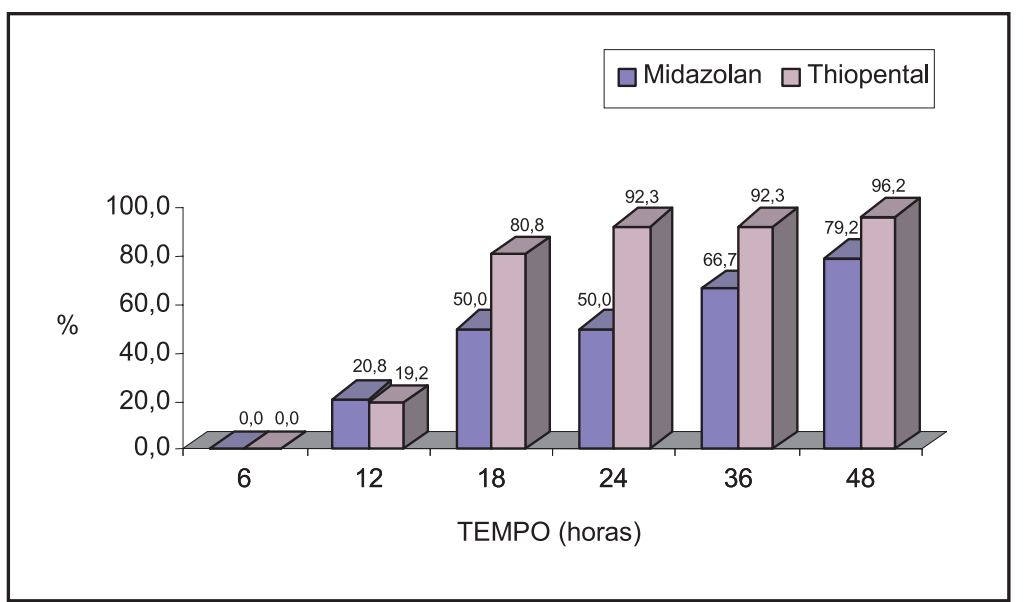

Fig 3. Controle das crises observadas nos pacientes dos grupos de midazolam e de thiopental.

Shanahan ${ }^{14}$, analisando 193 crianças com EME, constataram predomínio de 2/3 dos casos para o gênero masculino, enquanto, Errikson \& Koivikko ${ }^{15}$ e Maytal e col $^{16}$ não encontraram esta diferença. O fator causal prevalente do EME nos dois grupos foi o predominantemente sintomático; no Grupo Midazolam, a prevalência recaiu sobre a etiologia sintomática remota (pacientes com atraso psicomotor associados à história de epilepsia). e no Grupo Thiopental a etiologia sintomática aguda predominou. Estes achados são coincidentes com os apontados na literatura ${ }^{3,17,18}$.

Com relação aos tipos de crises, no Grupo Midazolam a somatória dos tipos de crises foi relativamente igual, ou seja, a metade dos pacientes apresentou EME por crises parciais e o restante, crises generalizadas. Esses dados são semelhantes aos encontrados por Garzon e col. ${ }^{19}$. No Grupo Thiopental predominaram as crises generalizadas, assemelhando-se a Eriksson \& Koivikko ${ }^{15}$.

A necessidade de ventilação mecânica foi significativamente maior no Grupo Thiopental, em decorrência da utilização de droga barbitúrica. A maioria dos pacientes do Grupo Midazolam não necessitou de ventilação assistida por apresentarem menos sedação e menos ação depressora que o thiopental, fato este já relatado ${ }^{6,7}$. Nas primeiras 6 horas foram observadas recorrência de convulsões nos dois grupos, porém após as primeiras 24 horas observou-se controle relativamente melhor das crises no Grupo Thiopental. Nas 36 e 48 horas, o controle das crises foi semelhante nos dois grupos, porém com leve predomínio de controle para o Grupo Thiopental. Não houve, portanto, diferença estatística para os dois grupos, com relação ao tempo de controle das convulsões. Rivera et al. ${ }^{6}$, observaram um tempo médio de 0,78 horas (tempo entre 15 minutos e 240 minutos) para o controle das convulsões. Esses índices são semelhantes aos de Koul e col. ${ }^{7}$, que observaram um tempo médio para o controle das crises de 64,6 minutos. No presente estudo, o tempo de observação das crises foi em intervalos de 6 horas, motivo pelo qual, para efeito de tempo nas primeiras 6 horas, não houve controle total das crises. A dose de infusão contínua média intravenosa empregada de midazolam, para o controle das convulsões foi $2,0 \mu \mathrm{g} / \mathrm{kg} / \mathrm{min}$., dado semelhante aos de Rivera e col. ${ }^{6}$ e Koul e col. ${ }^{7}$, mas deve-se levar em consideração que no presente estudo 87,5\% dos pacientes do Grupo Midazolam estavam recebendo outros anticonvulsivantes simultaneamente. Por este motivo, a dose de infusão média eficaz do presente estudo, embora semelhante aos estudos da literatura ${ }^{6,7}$, não pode ser considerada idêntica. A taxa de mortalidade por EME nos dois grupos foi similar, e próxima da cifra encontrada por Garzon e col. ${ }^{19}$ e Garduño-Espinosa e col..$^{20}$, que registraram taxa de $19,8 \%$ e $12,8 \%$ respectivamente.

O aparecimento de inquietude, períodos de agressividade, desorientação, risos não motivados e alucinação no Grupo Midazolam, coincidem com as observações relatadas por Sury e col. ${ }^{21}$, e Hughes e col. ${ }^{22}$. Estes autores, descreveram muito bem que tais alterações foram encontradas em 
$17 \%$ dos pacientes submetidos à terapia de sedação contínua com o midazolam. Estes fenômenos não foram observados em crianças menores de 2 anos.

\section{CONCLUSÃO}

O midazolam mostrou-se eficaz no controle do EME em crianças, em todos os tipos de crises convulsivas associado a outros anticonvulsivantes. Não houve diferença estatisticamente significativa no tratamento nos dois grupos com relação ao tempo, embora no Grupo Thiopental o controle tenha sido sensivelmente melhor nas primeiras 24 horas.

O valor médio da dose de infusão contínua foi semelhante ao encontrado por outros autores. A maioria dos pacientes do Grupo Thiopental só recebeu esta droga, enquanto que no Grupo Midazolam geralmente recebiam diversas outras drogas. A maioria dos pacientes que usaram o midazolam não necessitaram de ventilação mecânica e apresentaram menos complicações respiratórias. O midazolam é uma droga que pode ser empregada no estado de mal epiléptico juntamente com outros anticonvulsivantes antes do thiopental, o qual deve ser utilizado nos casos refratários ao midazolam. Face à recorrência das crises em alguns pacientes e ao surgimento de alterações psíquicas em outros, especula-se que este medicamento, sob a forma de uso contínuo e prolongado, possa induzir mais rapidamente a tolerância e a dependência do que os demais benzodiazepínicos e os barbitúricos.

\section{REFERÊNCIAS}

1. Gastaut H. Classification of status epilepticus. Adv Neurol 1994;34:15-35.

2 Gurgueira GL. Estado de mal epiléptico. In Carvalho WB, et al. Cuidados neurológicos em terapia intensiva pediátrica. São Paulo: Editora Louise, 1998:93- 124.

3 Cockerell OC, Shorvon SD. Epilepsia: conceitos atuais. São Paulo: Lemos, 1997:38-47.

4 Tasker RC, Dean JM. Status epilepticus. In Rogers MC (ed). Textbook of pediatric intensive care. Baltimore: Williams \& Williams, 1992;751-777.

5 Hauser WA. Status epilepticus: epidemiologic considerations. Neurology 1990;40:9-13.

6 Rivera R, Segnini M, Baltodano A, Pérez V. Midazolam in the treatment of status epilepticus in children. Crit Care Med 1993;21:991-994.

7 Koul RL, Aithala GR, Chacko A, Joshi R, Elbualy MS. Continuous midazolam infusion as treatment of status epilepticus. Arch Dis Child, 1997;76:445-448.

8 Tunik MG, Young GM. Status epilepticus in children: the acute management. Pediatr Clin N Am 1992;39:1007-1030.

9 Galvin GM, Jelinek GA. Successful treatment of 75 pacients in status epilepticus with intravenous midazolam. Emerg Med Clin N Am1992;4:11-12.

10 Delgado-Escueta AV, Wasterlain C, Treiman DM, Porter RJ. Current concepts in neurology: management of status epilepticus. N Engl J Med 1982;306:1337-1340.

11 Katayama D, Marques-Dias MJ. Estado de mal epiléptico. In Wong A. Terapia intensiva em pediatria. São Paulo: Sarvier, 1982;127-130.

12 Orlowski TP, Rothner AD. Diagnosis and treatment of status epilepticus. In Zimmerman JJ. (ed). Pediatric critical care. St. Louis: Mosby Year Book. 1992:595-604.

13 Shinnar S, Maytal J, Krasnoff L, Moshé S. Recurrent status epilepticus in children. Ann Neurol 1992;31:598-604.

14 Phillips AS, Shanahan RJ. Etiology and mortality of status epilepticus in children: a recent update. Arch Neurol 1989;46:74-76.

15 Eriksson KJ, Koivikko MJ. Status epilepticus in children: aetiology, treatment, and outcome. Dev Med Child Neurol 1997;39:652-658.

16 Maytal J, Shinnar S, Moshé SL, Alvares LA. Low morbidity and mortality of status epilepticus in children. Pediatrics 1989;83:323-331.

17 Aicardi J, Chevrie JJ. Convulsive status epilepticus in infants and children. Epilepsia 1970;11:187-197.

18 Aminoff MT, Simon RP. Status epilepticus: causes clinical features and consequences in 98 patients. Am J Med 1980;69:657-666.

19 Garzon E, Sakamoto A C, Fernandes RMF, Guerreiro CAM. Estado de mal epiléptico In Costa JC (ed). Fundamentos neurobiológicos das epilepsias-aspectos clínicos e cirúrgicos. São Paulo: Lemos Editorial 1998:417-443.

20 Garduño-Espinosa A, González-Astiazarán, Calvo-Gonzalez MS, Vaderábano-Ojeda L, Maulen-Radovan I. Estado epiléptico en niños: estudio de 70 casos. Bol Med Hosp Infant Mex 1990;47:567-575.

21 Sury MRJ, Billingham I, Russell GN, Hopkins CS, Thorington R, Vivori E. Acute benzodiazepine withdrawal syndrome after midazolam Infusions in children. Crit Care Med 1989;17:301-302.

22 Hughes J, Gill A, Leach HJ, et al.. A prospective study of the adverse effects of midazolam on withdrawal in critically ill children. Acta Paediatr 1994;83:1194-1199. 